\title{
The comet disintegration and meteor streams.
}

\author{
A. S. Guliyev and U. J. Poladova \\ Shamakha Astrophysical Observatory (www.shao.az), Azerbaijan, \\ email: ayyub54@yahoo.com
}

Possibility of disintegration of proto-comet nucleus of sungraser comets in three zones of Solar System predicted by one of authors is considered. Testing of parameters of 118 split comets confirms the basic idea. Results of the statistical analysis of comet outbursts gave us additional argument in favor of this assumption. Almost twenty years have passed since, as a result of the search for host phases of isotopically unusual noble gases, the first discovery in 1987 of surviving pre-solar minerals (diamond and silicon carbide) in primitive meteorites. These were followed by others (graphite, refractory oxides, silicon nitride, and finally silicates) in the years since. Pre-solar grains occur in even higher abundance than in meteorites in interplanetary dust particles (IDPs). The result is a kind of 'new astronomy' based on the study of pre-solar condensates with all the methods available in modern analytical laboratories. According to Guliev's hypothesis (Guliyev (2010)) comet families of Kreutz, Meyer and Kracht have been appeared regarding to falling of three proto-comet nuclear into hypothetical meteor streams in zones $\left(I_{p}=\right.$ $76^{0} .34 ; \Omega_{p}=267^{0} .15 ; 1.5$ a.u. $\leqslant r_{1} \leqslant 2.5$ a.u. $) ;\left(I_{p}=84^{0} .68 ; \Omega_{p}=106^{0} .03 ; 1.5 a . u . \leqslant\right.$ $r_{2} \leqslant 2.5$ a.u. $)$ and $\left(I_{p}=14^{0} .93 ; \Omega_{p}=54^{0} .26 ; 0.4 a . u . \leqslant r_{3} \leqslant 0.6 a . u\right.$. $)$. These zones numbered as 1,2 and 3. We will try to find out is there are overpopulation of nearest and distant nodes of other splitted comets and having outburst ones in zones (1-3). Data of 118 split comets discovered up to 2012 have been used in our work. We have calculated the numbers of distant nodes for all 118 comets relative to the selected planes (1-3) and found number of nodes $(\mathrm{N})$ corresponding to the interval $\left(r_{1}\right.$ and $\left.r_{2}\right)$ from zones $1-3$. We have also used the method of testing (Guliyev 2010) to demonstrate of excessiveness of N. On the base of data regarding to 67 comparison planes we have determined parameters: $\mathrm{n}$ - midrange value of nodes; $\sigma$ - rms deviation; $\mathrm{t}$ - normalized difference $(\mathrm{t}=(\mathrm{N}-\mathrm{n}) / \sigma)$ and $\alpha$ - confidential probability of t. Calculation and analyze of distant nodes regarding to the zone 1,2 and 3 gave us following numerical results: Zone 1 - distant nodes: $\mathrm{N}=20$; $\mathrm{n}=14.4478 ; \sigma=3.87 ; \mathrm{t}=1.43 ; \alpha=0.85$. Zone 1 - nearest nodes: $\mathrm{N}=29 ; \mathrm{n}=22.63 ; \sigma=3.54$; $\mathrm{t}=2.36 ; \alpha=0.99$. Zone 2 - distant nodes: $\mathrm{N}=15 ; \mathrm{n}=11.36 ; \sigma=3.02 ; \mathrm{t}=1.21 ; \alpha=0.75$. Zone 2 - nearest nodes $\mathrm{N}=40 ; \mathrm{n}=34.72 ; \sigma=2.71 ; \mathrm{t}=1.95 ; \alpha=0.97$. Zone 3 - nearest nodes $\mathrm{N}=9 ; \mathrm{n}=5.64 ; \sigma=1.49 ; \mathrm{t}=2.24 ; \alpha=0.99$. Zone 3 - distant nodes $\mathrm{N}=3 ; \mathrm{n}=0.49 ; \sigma$ $=0.75 ; \mathrm{t}=3.36 ; \alpha=0.99$. Thus in all three cases we have obtained confirmation of excess of comet nodes in zones (1-3). In zones 1,2 and 3 number of comet outbursts have to be more than in other ones. For checking of this consequence we demonstrate below (Fig.1. and Fig.2.)distribution of $\mathrm{q}$ of long-period comets and $\mathrm{r}$ (distant where comet outbursts have been observed), which confirms basis idea of (Guliyev (2010)).

\section{Reference}

Guliyev, A. S. 2010, Origin of short-perihelion comets.Publishing company,"Elm", Baku, 151 


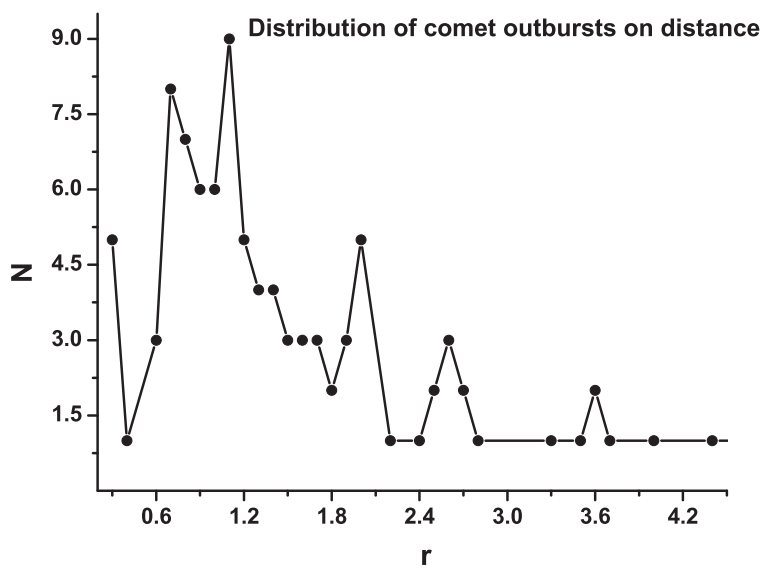

Figure 1. Distribution of comet outbursts as a function of distance from the Sun.

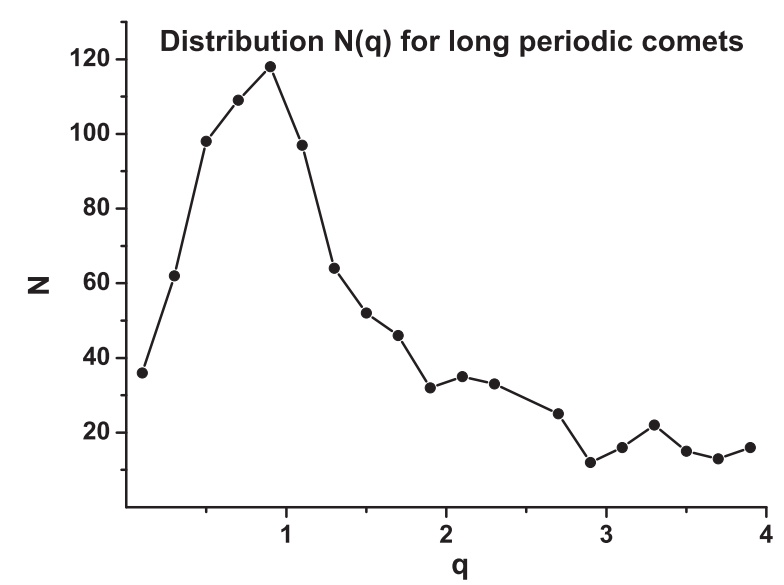

Figure 2. Distribution $\mathrm{N}(\mathrm{q})$ for long periodic comets. $\mathrm{q}$ is perihelion distance. 